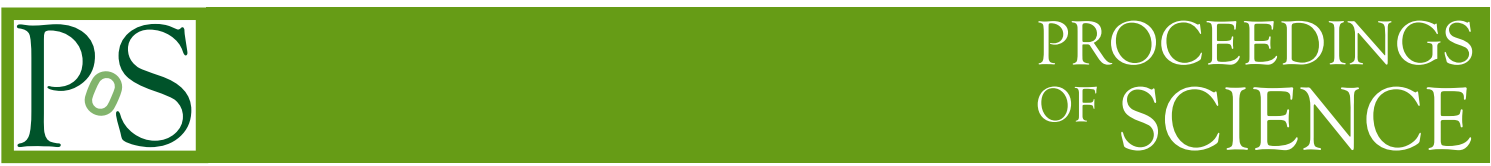

\title{
Higgs at last. And now what?
}

\author{
Adam Falkowski \\ Laboratoire de Physique Théorique d'Orsay, UMR8627-CNRS, \\ Université Paris-Sud, Orsay, France. \\ E-mail: afalkows017@gmail.com
}

\section{Francesco Riva}

Institut de Théorie des Phénomènes Physiques, EPFL,1015 Lausanne, Switzerland.

E-mail: Francesco.Riva@cern.ch

\section{Alfredo Urbano*}

SISSA - International School for Advanced Studies, via Bonomea 265, I-34136 Trieste, ITALY.

E-mail: alfredo.urbano@sissa.it

In the Standard Model the appearance of the Higgs boson is marked by three distinctive features: $i$ ) the spin and parity quantum numbers, $J^{P}=0^{+}$, ii) the self-coupling, proportional to its mass, and iii) the couplings with the electroweak gauge bosons and fermions, proportional to their masses. On the one hand, the value of the spin has been widely confirmed by the experimental analysis, with a strong preference for positive parity. On the other one, the measurement of the quartic Higgs self-coupling, accessible only in double Higgs production, represents a challenging goal to be pursued with higher luminosities. The Higgs couplings with gauge bosons and fermions, on the contrary, are currently under investigation at the LHC, and a precise knowledge of their values is crucial in order to establish if and where effects of new physics are present. We examine from a phenomenological viewpoint the most relevant aspects of this research, highlighting the places where deviations from the Standard Model may appear in the next future.

The European Physical Society Conference on High Energy Physics -EPS-HEP2013

18-24 July 2013

Stockholm, Sweden

\footnotetext{
*Speaker.
} 


\section{Introduction}

The effective field theory is an economical framework for interpreting the experimental data related to the Higgs couplings. Following Ref. [1], we start from five assumptions: $i$ ) the resonance experimentally observed near $126 \mathrm{GeV}$ is a color-neutral scalar particle $h$ with positive parity, ii) there are no light degrees of freedom, besides the SM particles, significantly coupled to the Higgs, iii) there are no flavor-violating Higgs couplings, $i v$ ) the Higgs couplings preserve the custodial symmetry, under which $h$ is a singlet, and $v$ ) there are no power-like divergences in the electroweak (EW) observables. The effective Lagrangian corresponding to this setup is

$$
\begin{aligned}
\mathscr{L}_{\mathrm{LO}} & =\frac{h}{v}\left[c_{V}\left(2 m_{W}^{2} W_{\mu}^{+} W^{-\mu}+m_{Z}^{2} Z_{\mu} Z^{\mu}\right)-c_{t} \sum_{f=u, c, t} m_{f} \bar{f} f-c_{b} \sum_{f=d, s, b} m_{f} \bar{f} f-c_{\tau} \sum_{f=e, \mu, \tau} m_{f} \bar{f} f\right], \\
\mathscr{L}_{\mathrm{NLO}} & =-\frac{h}{4 v}\left(2 c_{Z \gamma} A_{\mu v} Z^{\mu v}+c_{\gamma \gamma} A_{\mu v} A^{\mu v}-c_{g g} G_{\mu \nu}^{A} G^{A \mu v}\right),
\end{aligned}
$$

with $v \approx 246 \mathrm{GeV}$. The SM corresponds to $c_{V}=c_{t}=c_{b}=c_{\tau}=1, c_{\gamma \gamma}=c_{Z \gamma}=c_{g g}=0$. The effects of new physics, therefore, are encoded in possible deviations of the leading order (LO) Higgs couplings from their SM values, and in the appearance of next-to-leading order (NLO) interactions. All in all, as a consequence, we have only 7 free parameters, namely $c_{V}, c_{t}, c_{b}, c_{\tau}$ at $\mathrm{LO}$, and $c_{\gamma \gamma}, c_{Z \gamma}, c_{g g}$ at NLO. We refer to Ref. [1] for a more detailed discussion.

\section{The LEP legacy}

The legacy of the LEP experiment is contained in the definition of the oblique parameters, i.e. the radiative corrections that alter the vacuum polarization amplitudes of the EW gauge bosons. All the experimental data collected during both the LEP-I and LEP-II runs, in fact, can be analyzed in terms of three independent EW observables, $S, T, U$, and their higher-order extension $V, W, X$, $Y$. In the setup described by the effective Lagrangian in Eq. (1.1) we find

$$
\begin{aligned}
\alpha S & =\frac{g_{\mathrm{Y}} g_{\mathrm{L}}}{48 \pi^{2}\left(g_{\mathrm{L}}^{2}+g_{\mathrm{Y}}^{2}\right)}\left\{2 g_{\mathrm{Y}} g_{\mathrm{L}}\left(1-c_{V}^{2}\right)+6 c_{V}\left[2 g_{\mathrm{Y}} g_{\mathrm{L}} c_{\gamma \gamma}+c_{Z \gamma}\left(g_{\mathrm{L}}^{2}+g_{\mathrm{Y}}^{2}\right)\right]\right\} \ln \frac{\Lambda}{m_{\mathrm{Z}}}, \\
\alpha T & =\frac{3 g_{\mathrm{Y}}^{2}}{32 \pi^{2}}\left(c_{V}^{2}-1\right) \ln \frac{\Lambda}{m_{\mathrm{Z}}} ;
\end{aligned}
$$

the parameters $U, V, X$ are not logarithmically enhanced, while $W$ and $Y$ receive corrections of or$\operatorname{der} \mathscr{O}\left(c_{Z \gamma}^{2}, c_{\gamma \gamma}^{2}\right)$, beyond the adopted NLO approximation. The oblique corrections in Eqs. $(2.1,2.2)$ are strongly constrained by the LEP data. To be more quantitative, we fit all the EW precision data according to Table 1 in Ref. [1], and we show the allowed ellipses for $S$ and $T$ in the left panel of Fig. 1. The reference point at which all oblique parameters vanish is defined by the SM with $m_{h}=126 \mathrm{GeV}$ and $m_{t}=173.5 \mathrm{GeV}$, and it lies on the boundary of the $68 \%$ confidence contour, in perfect agreement with the experimental data. Allowing for deviations of the Higgs couplings it is possible to move away from this point, as shown by the representative arrows in the plot. The LEP fit, as a consequence, can be translated into bounds on $c_{V}, c_{Z \gamma}, c_{\gamma \gamma}$. We perform a one-dimensional fit using $c_{V}$ as a free parameter, marginalizing over the remaining ones, and we show our result in the right panel of Fig. 1 (red dot-dashed line). We find $c_{V}=1.07_{-0.13}^{+0.12}$ at $95 \%$ C.L., thus already 

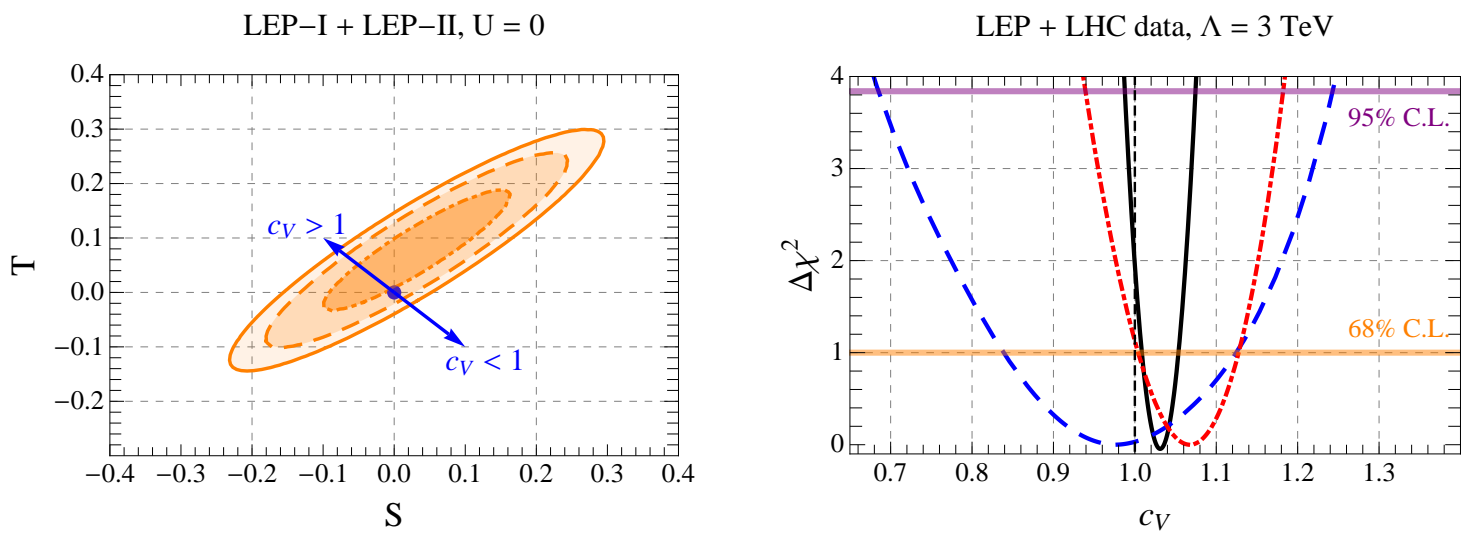

Figure 1: Left panel, fit $(68 \%, 95 \%$, and $99 \%$ C.L.) of the LEP data in the $S T$-plane, with $U=0$. Right panel, fit of the LEP and LHC data w.r.t. the Higgs couplings $c_{V}$. Red dot-dashed line: only LEP. Blue dashed line: only LHC. Black solid line: LEP+LHC. In the EW data we also include the $m_{W}$ measurement at the SLC.

providing a strong constraint on the value of the tree level Higgs coupling with $W, Z$. Two caveats are mandatory. First, the constraints depend logarithmically on the cut-off scale of the effective field theory, here chosen at $\Lambda=3 \mathrm{TeV}$. Second, the constraints can go away counterbalancing the Higgs contributions with other significant corrections coming from new physics interactions complementary to the Higgs effective Lagrangian in Eq. (1.1).

\section{Higgs couplings at the $\mathrm{LHC}$}

We compute the Higgs branching ratios and production cross sections using Eq. (1.1), obtaining the corresponding expressions as a function of the parameters in the effective Lagrangian, $\sigma_{p}\left(c_{\mathrm{LO}}, c_{\mathrm{NLO}}\right), \mathrm{BR}_{i}\left(c_{\mathrm{LO}}, c_{\mathrm{NLO}}\right)$. The production mechanisms $\sigma_{p}$ are ggF (gluon fusion), $\mathrm{VBF}$ (vector boson fusion), $\mathrm{VH}$ (vector boson associated production), and ttH (top quark associated production). The branching ratios $\mathrm{BR}_{i}$ are related to the Higgs decay channels under investigation at the LHC, $h \rightarrow Z Z^{*} \rightarrow 4 l^{ \pm}, h \rightarrow W W^{*} \rightarrow 2 l 2 v, h \rightarrow \gamma \gamma, h \rightarrow \tau^{+} \tau^{-}, h \rightarrow b \bar{b}$. The theoretical prediction for the signal strengths $\hat{\mu}_{i}$ is therefore given by

$$
\hat{\mu}_{i}=\left[\frac{\sum_{p} \sigma_{p}\left(c_{\mathrm{LO}}, c_{\mathrm{NLO}}\right) \zeta_{p}^{i}}{\sum_{p} \sigma_{p}^{\mathrm{SM}} \zeta_{p}^{i}}\right] \times \frac{\mathrm{BR}_{i}\left(c_{\mathrm{LO}}, c_{\mathrm{NLO}}\right)}{\mathrm{BR}_{i}^{\mathrm{SM}}},
$$

where the sum runs over the production mechanisms while $\zeta_{p}^{i}$ are the corresponding cut efficiencies. Using this formula we perform a $\chi$-square fit of the signal strengths measured at ATLAS and CMS, according to the data collected in Table 2 in Ref. [1]. As a first exercise, we repeat the onedimensional fit on $c_{V}$, combining LHC and LEP data, and we show our result in the right panel of Fig. 1 (black line). We find $c_{V}=1.03_{-0.04}^{+0.03}$ at $95 \%$ C.L. $\left(c_{V}=0.97_{-0.29}^{+0.27}\right.$ considering only the LHC data, blue dashed line), thus confirming the $\mathrm{SM}$ value $c_{V}=1$ with great statistical significance. Let us now consider some relevant analysis in the context of beyond the SM physics.

$*$ LOOP NEW PHYSICS. We define $\delta c_{\gamma \gamma} \equiv c_{\gamma \gamma}-c_{\gamma \gamma}^{\mathrm{SM}}, \delta c_{Z \gamma} \equiv c_{Z \gamma}-c_{Z \gamma}^{\mathrm{SM}}$, and we perform a two-dimensional fit in the plane $\left(\delta c_{\gamma \gamma}, \delta c_{Z \gamma}\right)$, setting all the other Higgs couplings to zero (see Fig. 2, left panel). The aim of this analysis is to quantify the possibility that new colorless particles 
modify the loop-induced Higgs couplings with $\gamma \gamma$ and $Z \gamma$. On the one hand we obtain a quite strong limit on the coupling with photons (c.f. $c_{\gamma \gamma}^{\mathrm{SM}}=0.0076$ ), but a weak limit on the coupling with $Z \gamma$, due to poor experimental precision (c.f. $c_{Z \gamma}^{\mathrm{SM}}=0.014$ ). We also show the contour of constant enhancement for the ratio $\Gamma_{Z \gamma} / \Gamma_{Z \gamma}^{\mathrm{SM}}$ (red dashed line) and $\sigma_{W H} / \sigma_{W H}^{\mathrm{SM}}$ (blue dotted line). Large deviations from the SM values are perfectly allowed by the present experimental constraints.
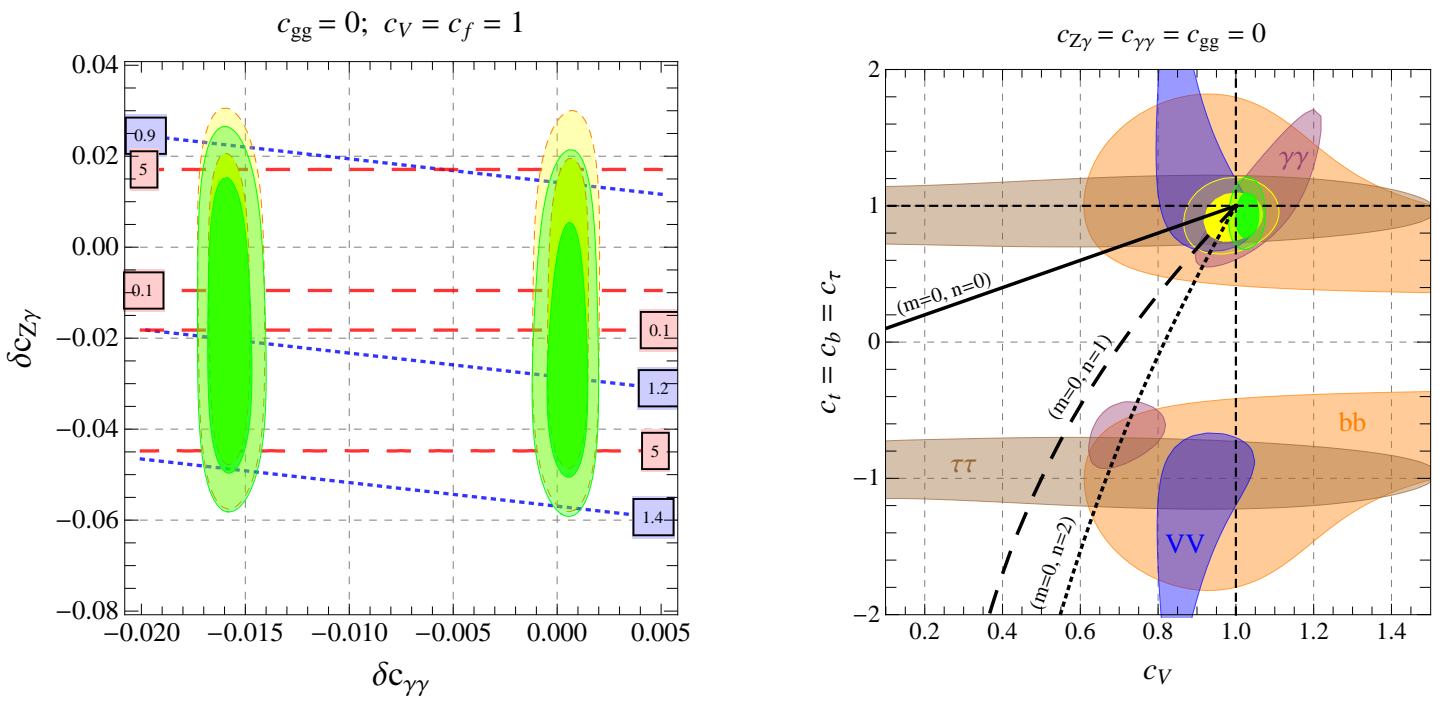

Figure 2: Left panel: two-dimensional fit (68\% and 95\% C.L.) in the plane $\left(c_{\gamma \gamma}, c_{Z \gamma}\right)$. Right panel: twodimensional fit (68\% C.L. for each channel, $68 \%$ and $95 \%$ C.L. in combination) in the plane $\left(c_{V}, c_{f}\right)$. Yellow: LHC. Green: LHC+LEP. Black lines describe the deviations predicted by CHM [2].

* COMPOSITE HIGGS. In Composite Higgs Models (CHM) the Higgs is a pseudo NambuGoldstone boson emerging from a global symmetry spontaneously broken at a new scale $f$ by a strong dynamics, and explicitly broken by the electroweak interactions. At low energy this theoretical setup leaves a mark modifying the tree-level Higgs couplings with gauge bosons $\left(c_{V}=\right.$ $\sqrt{1-v^{2} / f^{2}}$ ) and fermions (the explicit expression for the coupling $c_{f}$ with fermions is modeldependent [2]). We perform a two-dimensional fit in the plane $\left(c_{V}, c_{f}\right)$, setting all the other parameters to zero (see Fig. 2, right panel). Data show a strong preference for the SM point. In the left panel of Fig. 3 we show the one-dimensional fit w.r.t. the compositeness scale $f$. Considering only LHC data, we find that $f \lesssim 600 \mathrm{GeV}$ is excluded at $2 \sigma$ level $(f \lesssim 1.2 \mathrm{TeV}$ including LEP data. Notice, however, that extra contributions from the strong sector can weaken this bound).

* INVISIBLE HIGGS DECAY. We add as an additional parameter the Higgs decay into invisible particles; this scenario is motivated by the interplay between Higgs and dark matter. First, we perform a one-dimensional fit w.r.t. the invisible Higgs branching ratio, $\mathrm{BR}_{\mathrm{inv}}$, setting all the couplings to their SM values. We show our result in the right panel of Fig. 3, where we find that $\mathrm{BR}_{\mathrm{inv}} \gtrsim 23 \%$ is disfavored at $2 \sigma$ level. However, it is important to keep in mind that a weaker bound can be obtained allowing for deviations of the NLO Higgs couplings. Marginalizing over $c_{g g}, c_{\gamma \gamma}$ for instance, we find that $\mathrm{BR}_{\mathrm{inv}} \gtrsim 50 \%$ is excluded at $2 \sigma$ level.

$*$ COMPARISON WITH ATLAS AND CMS. Finally, in Fig. 4 we compare our results with the fit performed by the experimental collaborations [3, 4]. We find a decent agreement, despite 

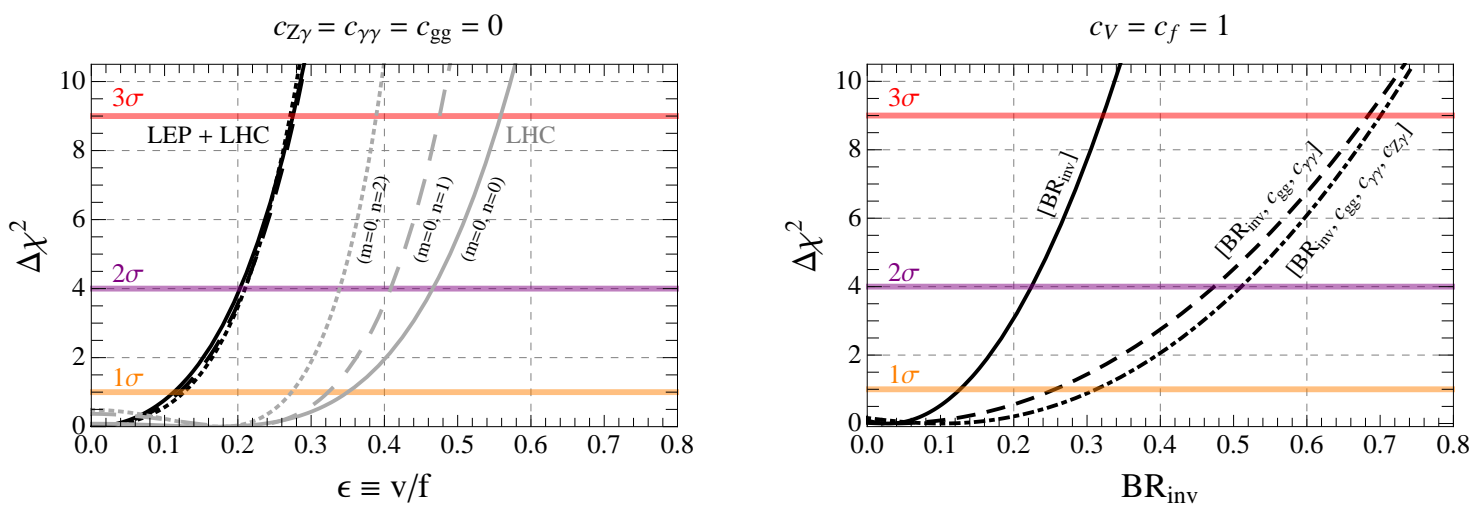

Figure 3: Left panel: one-dimensional fit w.r.t. the compositeness scale $f$. Right panel: one-dimensional fit w.r.t. the invisible branching ratio of the Higgs.
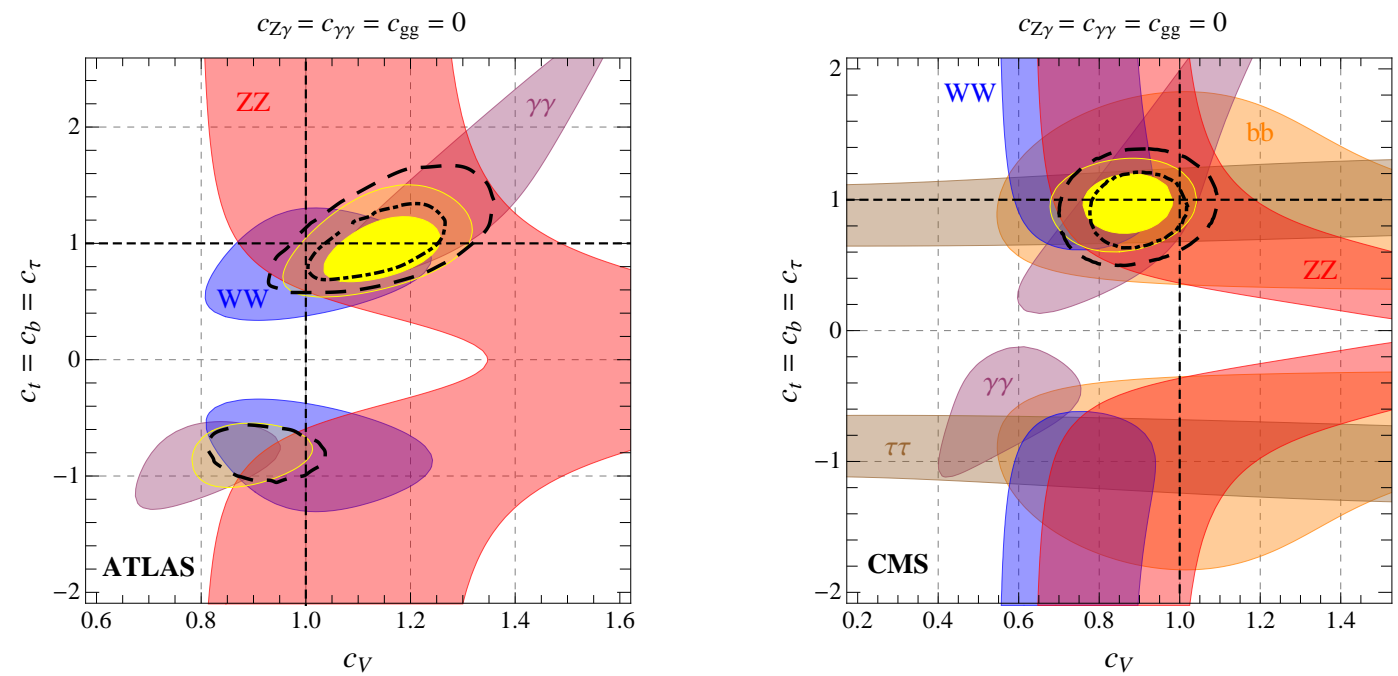

Figure 4: Comparison with the fit performed by ATLAS and CMS.

the fact that our approach ignores systematics and correlations.

\section{Conclusions and outlook}

In conclusion, we have analyzed all the Higgs data collected at the LHC parametrizing the Higgs couplings with SM fields using an effective field theory language. Large deviations in the $h \rightarrow Z \gamma$ decay channel, as well a a sizable invisible branching ratio are still possible.

\section{References}

[1] A. Falkowski, F. Riva and A. Urbano, Higgs At Last, arXiv:1303.1812.

[2] A. Pomarol and F. Riva, The Composite Higgs and Light Resonance Connection, JHEP 1208, 135 (2012) [arXiv:1205.6434].

[3] [ATLAS Collaboration], ATLAS-CONF-2013-034.

[4] [CMS Collaboration], CMS-PAS-HIG-13-005. 